\title{
Intelligent System on Chip for Aircraft Landing Control
}

\author{
Jhen-Tang Dai, Chia-Ling Lee, and Jih-Gau Juang* \\ Department of Communications, Navigation and Control Engineering, National Taiwan Ocean University, \\ 2 Pei-Ning Road, Keelung, 20224, Taiwan
}

(Received April 12, 2021; accepted November 25, 2021)

Keywords: PSO, AWPSO, GPSO, SMC, ALS, wind shear

We propose sliding mode control (SMC) with different types of particle swarm optimization for an aircraft automatic landing system (ALS). The controller parameters are adjusted by a fuzzy system, adaptive weight particle swarm optimization, and grey-based particle swarm optimization. When an aircraft encounters wind shear, it diverges from the originally scheduled flight condition, and the aircraft ALS cannot be used in such an environment during large wind speed changes. The proposed intelligent control scheme can help pilots guide an aircraft to a safe landing in wind shear of up to $58 \mathrm{ft} / \mathrm{s}$. In addition to software simulation, real-time automatic landing control is realized by an embedded floating-point digital signal processor (DSP). Control signals are obtained by relative sensors and sent to the DSP in a hardware simulation.

\section{Introduction}

On March 1, 2008, a Lufthansa Airbus A320 tried to land at Hamburg airport in a crosswind that exceeded the limit for the aircraft, causing the left wing to touch the ground. The pilots then performed a go around and successfully prevented the aircraft from crashing. This case is a successful example of pilot skill. However, junior pilots might not be able to save an aircraft in such a situation. It is well known that atmospheric disturbances cause flight safety problems and reduce the quality of flight for passengers. An accident survey(1) categorized the causes of 1085 aircraft accidents from 1950 through 2019, as shown in Table 1. Weather was a contributing factor for $10 \%$ of accidents. The first automatic landing system (ALS) was developed in the UK in 1965. Since then, most aircraft have had an ALS installed. The ALS relies on an instrument landing system to guide the aircraft to the proper altitude, position, and angle of approach during the landing phase. According to Federal Aviation Administration (FAA) regulations, ${ }^{(2)}$ the allowable environmental conditions in the determination of dispersion limits are headwinds of up to $25 \mathrm{knots}(1 \mathrm{knot}=1.852 \mathrm{~km} / \mathrm{h})$, tailwinds of up to $10 \mathrm{knots}$, crosswinds of up to $15 \mathrm{knots}$, moderate turbulence, and wind shear of 8 knots per 100 feet from 200 feet to touchdown. Conventional ALSs can provide a smooth landing, which is essential for the comfort of passengers. However, these systems work only within a specified operational safety envelope. When the conditions, such as turbulence or wind shear, are beyond the envelope, ALSs often cannot be used. Most conventional control laws generated by an ALS are based on the gain

*Corresponding author: e-mail: jgjuang@ntou.edu.tw

https://doi.org/10.18494/SAM.2022.3547 
Table 1

Causes of fatal accidents by decade from 1950 through 2019.

\begin{tabular}{lcccccccc}
\hline Decade & $1950 \mathrm{~s} \mathrm{( \% )}$ & 1960s (\%) & 1970s (\%) & 1980s (\%) & 1990s (\%) & 2000s (\%) & 2010s (\%) & All (\%) \\
\hline Pilot error & 50 & 53 & 49 & 42 & 49 & 50 & 57 & 49 \\
Mechanical & 26 & 27 & 19 & 22 & 22 & 23 & 21 & 23 \\
Weather & 15 & 7 & 10 & 14 & 7 & 8 & 10 & 10 \\
Sabotage & 4 & 4 & 9 & 12 & 8 & 9 & 8 & 8 \\
Other & 5 & 9 & 13 & 10 & 14 & 10 & 4 & 10 \\
\hline
\end{tabular}

scheduling method. ${ }^{(3)}$ Control parameters are preset for different flight conditions within a specified safety envelope, which is defined by the FAA regulations. When the conditions, such as wind shear, are beyond the specified flight envelope, the ALS is disabled and manual operation is engaged. Even low-level wind shear can affect aircraft airspeed during takeoff and landing in disastrous ways, and airline pilots are trained to avoid all wind shear. An inexperienced pilot may not be able to guide the aircraft safely under wind shear. Therefore, it is desirable to develop an intelligent ALS that expands the operational envelope to include more safe responses under a wider range of wind disturbance conditions. The goal of this study is to show that our proposed intelligent ALS can relieve human operation and guide the aircraft to a safe landing within a certain range of wind shear conditions.

In recent years, intelligent control has become increasingly popular in control engineering applications. Many intelligent concepts have been applied in various scientific and engineering research. There have also been major achievements in the flight control domain. ${ }^{(4-9)}$ In conventional control systems, proportional-integral-derivative (PID) control has been applied to controller design for decades and is the most commonly used type of control in engineering applications. The gains of PID control can be tuned by many techniques. In recent years, the genetic algorithm has been the most widely used technique.

In this study, we apply artificial life models for the modeling and simulation of lifelike phenomena to aircraft automatic landing controller design. This study is mainly based on two algorithms, which are used to adjust the control parameters of the pitch autopilot: adaptive weight particle swarm optimization (AWPSO) ${ }^{(10)}$ and grey-based particle swarm optimization (GPSO). ${ }^{(11)}$ Our control schemes are based on sliding mode control (SMC) with a fuzzy system (FSMC). ${ }^{(12-14)}$ To realize a hardware controller, a digital signal processor (DSP) is used and an intelligent aircraft landing control system is implemented on the chip. ${ }^{(15)}$ All signals are sensed by relative sensors and sent to the DSP in a hardware simulation. In our previous works, a DSP was successfully applied to a cerebellar model articulation controller for turbulence conditions. ${ }^{(15,16)}$ In this study, different PSO methods with FSMC on a chip are used to simulate the control of an aircraft landing under wind shear conditions.

\section{System Description}

In the aircraft landing phase, the pilot descends from the cruise altitude to an altitude of approximately $1200 \mathrm{ft}$ above the ground. The pilot then positions the aircraft so it is on a trajectory towards the runway centerline. When the aircraft approaches the outer airport marker, which is about 4 nautical miles from the runway, the glide path signal is intercepted, as shown in 
Fig. 1. ${ }^{(17)}$ As the airplane descends along the glide path, its pitch, attitude, and speed must be controlled. The descent rate is about $10 \mathrm{ft} / \mathrm{s}$ and the pitch angle is between $-5^{\circ}$ and $+5^{\circ}$. Finally, as the airplane descends to 20 to 70 feet above the ground, the glide path control system is disengaged and a flare maneuver is executed. The vertical descent rate is decreased to $2 \mathrm{ft} / \mathrm{s}$ so that the landing gear can dissipate the energy of the impact at landing. The pitch angle of the airplane is then adjusted to between $0^{\circ}$ and $5^{\circ}$ for most aircraft, which allows a soft touchdown on the runway surface.

A simplified model of a commercial aircraft that moves only in the longitudinal and vertical planes is used in the simulations for ease of implementation. ${ }^{(7)}$ The motion equations of the aircraft are given as follows:

$$
\begin{gathered}
\Delta \dot{u}=X_{u}\left(\Delta u-u_{g}\right)+X_{w}\left(\Delta w-w_{g}\right)+X_{q} \Delta q-g\left(\frac{\pi}{180}\right) \cos \left(\gamma_{0}\right) \Delta \theta+X_{E} \delta_{E}+X_{T} \delta_{T} \\
\Delta \dot{w}=Z_{u}\left(\Delta u-u_{g}\right)+Z_{w}\left(\Delta w-w_{g}\right)+\left(Z_{q}-\frac{\pi}{180} U_{0}\right) \Delta q-g\left(\frac{\pi}{180}\right) \sin \left(\gamma_{0}\right) \Delta \theta+Z_{E} \delta_{E}+Z_{T} \delta_{T} \\
\Delta \dot{q}=M_{u}\left(\Delta u-u_{g}\right)+M_{w}\left(\Delta w-w_{g}\right)+M_{q} \Delta q+M_{E} \delta_{E}+M_{T} \delta_{T} \\
\Delta \dot{\theta}=\Delta q \\
\Delta \dot{h}=-\Delta w+\frac{\pi}{180} U_{0} \Delta \theta
\end{gathered}
$$

where $u$ is the aircraft longitudinal velocity ( $\mathrm{ft} / \mathrm{s}), w$ is the aircraft vertical velocity (ft/s), $q$ is the pitch rate $(\%), \theta$ is the pitch angle $\left({ }^{\circ}\right), h$ is the aircraft altitude ( $\left.\mathrm{ft}\right), \delta_{E}$ is the incremental elevator angle $\left({ }^{\circ}\right), \delta_{T}$ is the throttle setting $(\mathrm{ft} / \mathrm{s}), \gamma_{o}$ is the flight path angle $\left(-3^{\circ}\right)$, and $g$ is acceleration due to gravity $\left(32.2 \mathrm{ft} / \mathrm{s}^{2}\right)$. The parameters $X_{i}, Z_{i}$, and $M_{i}$ are the stability and control derivatives.

In our previous study, the proposed method was successfully applied to turbulence conditions without hardware implementation. ${ }^{(14)}$ In this study, wind shear is considered as the disturbance, and the control system is implemented on a chip. In the presence of significant wind shear, a pilot has to take corrective action to ensure safety, as shown in Fig. 2. Figure 3 shows a wind shear profile for a wind speed of $60 \mathrm{ft} / \mathrm{s}$.

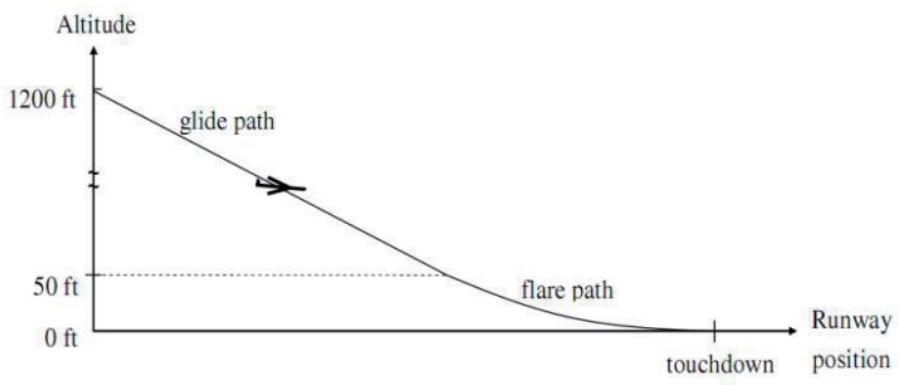

Fig. 1. Glide path and flare path. 


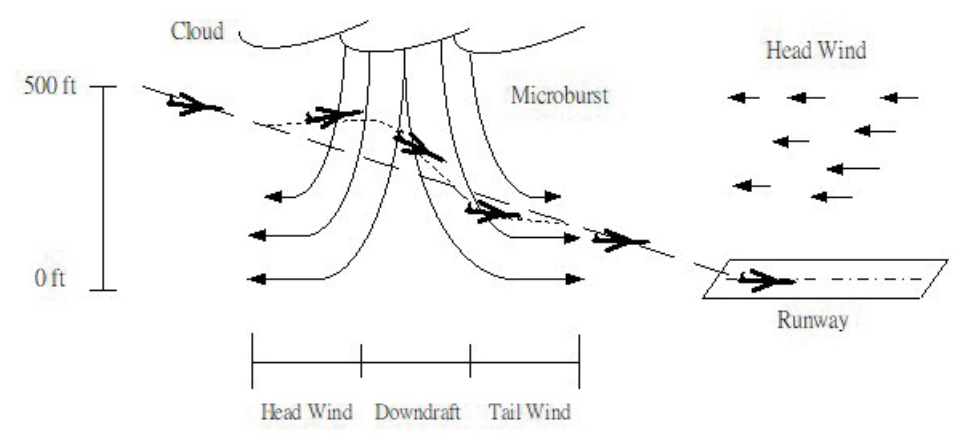

Fig. 2. Aircraft encountering wind shear.

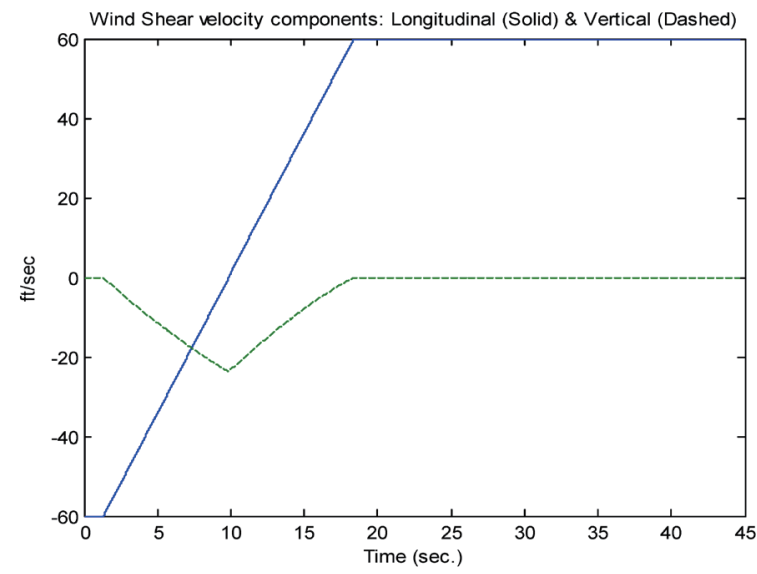

Fig. 3. (Color online) Profile of wind shear model.

\section{Intelligent Control}

A simplified PID controller is used in the aircraft landing control structure as shown in Fig. 4. Its inputs consist of altitude and altitude rate commands along with the aircraft altitude and altitude rate. We can obtain the pitch command via the aircraft landing controller. Then, the pitch autopilot is controlled by the pitch command. The pitch autopilot is shown in Fig. 5. Detailed descriptions can be found in Ref. 7. To enable an aircraft to land more stably when it reaches the flare path, a constant pitch angle is added to the controller. When severe turbulence is encountered, the PID controller may not be able to guide the aircraft to land safely. The proposed controller can overcome these disadvantages by employing an FSMC compensator, which uses a traditional PID controller to stabilize the system and FSMC to provide precise control. The gains of the FSMC controller are adjusted on the basis of experience to provide tolerable solutions rather than desired solutions. The FSMC can effectively meliorate these conditions.

The overall control scheme is shown in Fig. 6, in which the control signal $U$ is the sum of the PID controller output and the FSMC output. ${ }^{(14)}$ The inputs for the FSMC and PID controller are 


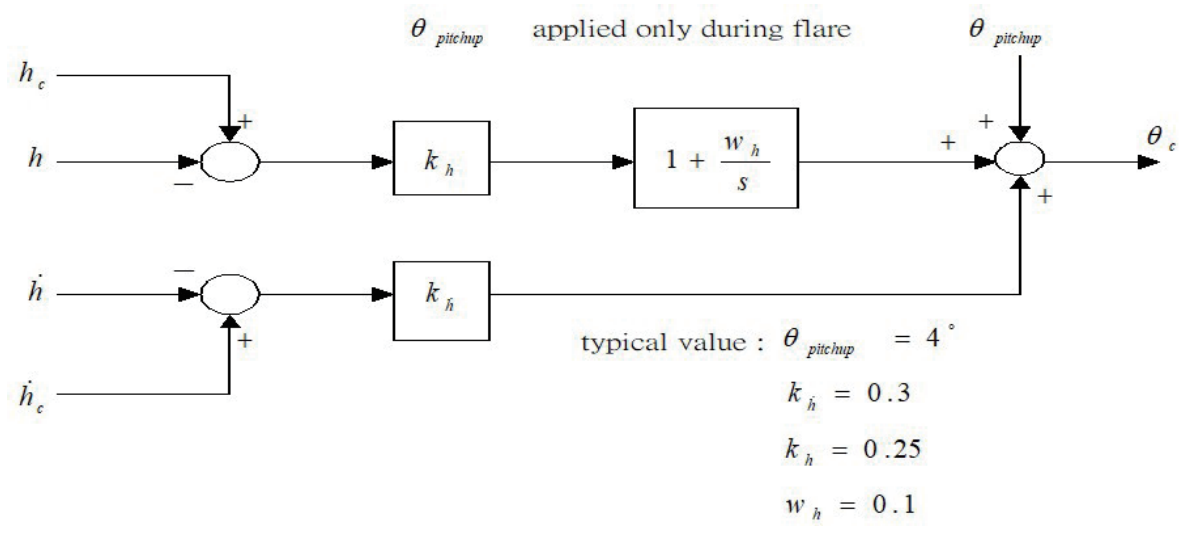

Fig. 4. PID controller.

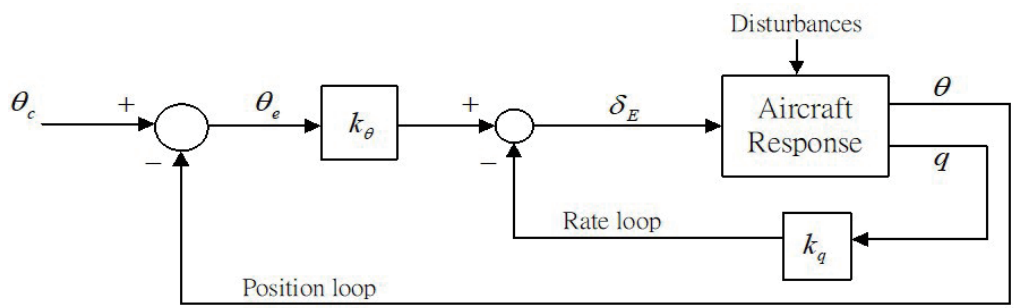

$$
\begin{aligned}
& \text { typical value-Glide Slope : } k_{\theta}=2.8 \\
& k_{q}=2.8 \\
& \text { Flare: } k_{\theta}=11.5 \\
& k_{q}=6.0
\end{aligned}
$$

Fig. 5. Pitch autopilot.

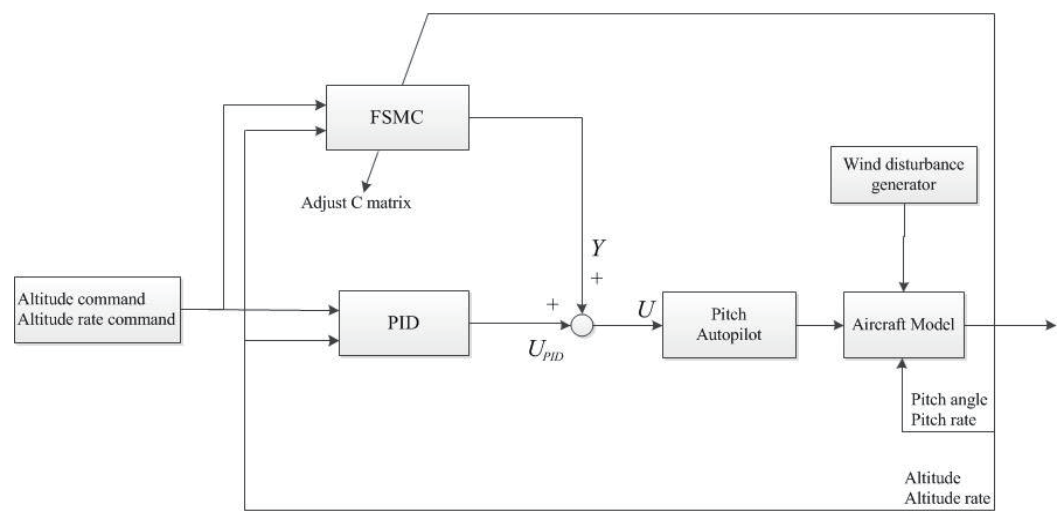

Fig. 6. Adjustment of control parameters and FSMC-PID structure.

altitude, altitude command, altitude rate, and altitude rate command. In each time step $k$, the FSMC involves a recall process and a learning process. In the recall process, it uses the desired system output of the next time step and the actual system output to generate the control signal $Y$. In the learning process, the control signal of the pitch autopilot, $U$, is treated as a desired output. The output of the FSMC compensates the pitch command. When the wind turbulence is too strong, the ALS cannot control the aircraft to make it land safely. Here, we use the proposed intelligent control scheme to improve the robustness of the ALS to turbulence. 


\subsection{SMC}

In general, the SMC design is divided into two main steps. The first step is to define the sliding surface. The second step is to design a control law such that the system will reach and proceed on the intersection of the sliding surface. ${ }^{(12-14)}$ The motion equations of the aircraft given in the previous section can be described by

$$
\dot{x}=A x+B u+D .
$$

The sliding surface is defined as

$$
s=C e,
$$

where $x \in \boldsymbol{R}^{5 \times 1}$ is the state vector, $u \in \boldsymbol{R}^{2 \times 1}$ is the input vector, $C \in \boldsymbol{R}^{2 \times 5}$ is the matrix of the controller parameters, $D \in \boldsymbol{R}^{5 \times 2}$ is the matrix of the disturbance, and $e \in \boldsymbol{R}^{5 \times 1}$ is the vector of the tracking error. Then the optimal equivalent control $u_{e q}$ can be obtained by solving

$$
\left.\dot{s}\right|_{u=u_{e q}}=0,
$$

From Eqs. (6) and (8), we have

$$
\begin{gathered}
\dot{s}=C \dot{e}=C\left(\dot{x}_{d}-\dot{x}\right)=C \dot{x}_{d}-C A x-C B u_{e q}=0, \\
u_{e q}=(C B)^{-1}\left(C \dot{x}_{d}-C A x\right) .
\end{gathered}
$$

We choose the switching control $u_{s w}$ as

$$
u_{s w}=(C B)^{-1} k \operatorname{sgn}(\mathrm{s})
$$

thus, the control law becomes

$$
u=u_{e q}+u_{s w}
$$

If the system state is outside the sliding surface, the controller must be designed such that it can force the system state to approach the sliding surface and then move along the sliding surface to the origin. By choosing the Lyapunov function as

$$
V=\frac{1}{2} s^{2},
$$


the derivative of Eq. (13) can be obtained as

$$
\dot{V}=s \dot{s}=-k|s|+C D s \leq(-k+|C D|)|s|<0,
$$

where $k$ is a positive constant. Thus, $\dot{V}<0$. Therefore, the control law given by Eq. (12) can guarantee that the sliding mode is reached and sustained.

Since SMC has a chattering phenomenon along the sliding surface, the sliding layer technique is utilized to eliminate the chattering of the input. The signum function $\operatorname{sgn}(s)$ is replaced by the function $\operatorname{sat}(s / \varepsilon)$, which is defined as

$$
\operatorname{sat}\left(\frac{s}{\varepsilon}\right)=\left\{\begin{array}{cc}
+1, & s>\varepsilon \\
\frac{s}{\varepsilon}, & -\varepsilon \leq s \leq \varepsilon \\
-1, & s<-\varepsilon
\end{array}\right.
$$

where $\varepsilon$ is a positive constant, as shown in Figs. 7 and 8.

\subsection{Fuzzy system}

For a stable control system, the derivative of the Lyapunov function [Eq. (14)] is less than zero, and the system states of the sliding mode should move along the sliding surface to the origin. In this study, FSMC is applied to acquire the controller gain $(k)$. In the FSMC, the control gain, and therefore $k$, is updated online and the stability of the system is guaranteed. Fuzzy control is an intelligent method that uses tentative rules to calculate the output variable. To use fuzzy control, we have to know the domain of the parameters and the fuzzy rule base. ${ }^{(13)}$ In Fig. $9, x$ is the input to the fuzzy system and $y$ is the output variable of the fuzzy system. We choose $s$ and $\dot{s}$ as the inputs of the fuzzy system to calculate $k$. The fuzzy rules are shown in Table 2 .

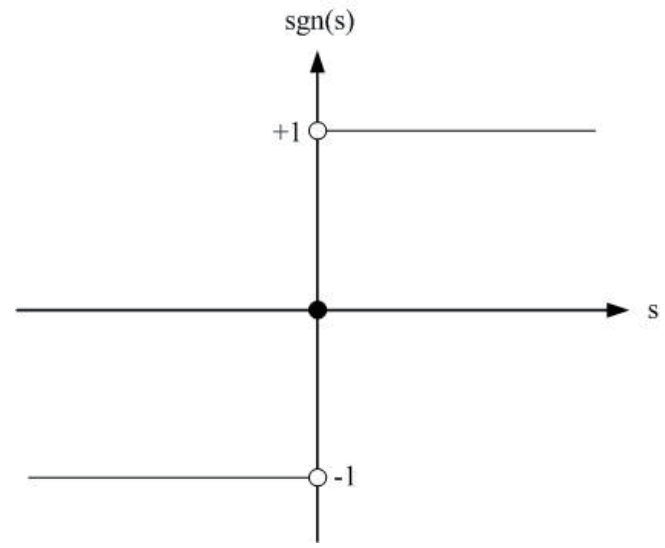

Fig. 7. Signum function.

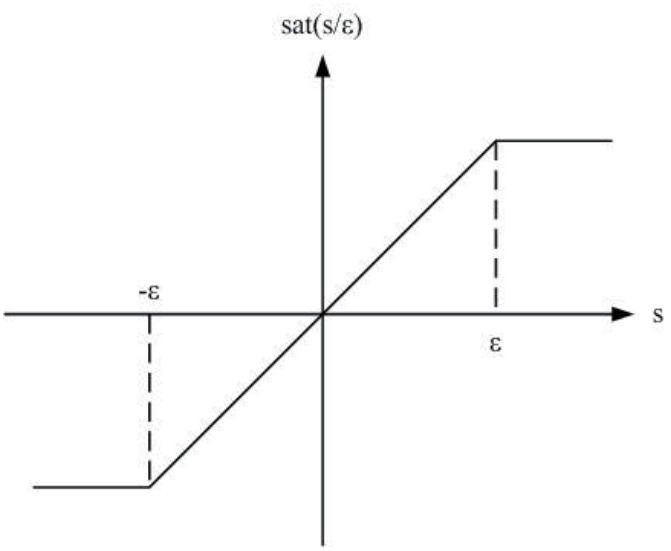

Fig. 8. Saturation function. 


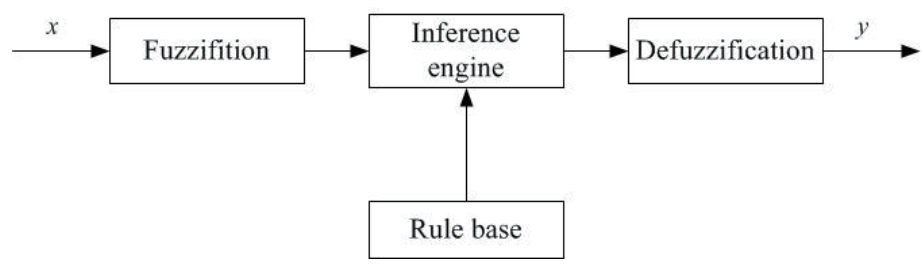

Fig. 9. Block diagram for a fuzzy system.

Table 2

Fuzzy rule table.

\begin{tabular}{ccccccc}
\hline$k$ & \multicolumn{7}{c}{$\mathrm{S}$} \\
\cline { 2 - 7 } & NB & NS & ZR & PS & PB & \\
\hline \multirow{4}{*}{$\dot{S}$} & PB & Z & S & M & B & B \\
& PS & S & Z & S & M & B \\
& ZR & M & S & Z & S & M \\
& NS & B & M & S & Z & S \\
& NB & B & B & M & S & Z \\
\hline
\end{tabular}

\subsection{Particle swarm optimization}

The main steps in the particle swarm optimization (PSO) process are described as follows. ${ }^{(18)}$

(i) Initialize a population (array) of particles with random positions and velocities in the problem space.

(ii) Calculate the fitness function [Eq. (16)], set the value to pbest for each particle, and set the best value of all the particles to gbest.

$$
f(x)=\left(u\left(x_{i d}^{(n)}\right)-180\right)^{-1}
$$

(iii) Change the velocity and position of each particle according to Eqs. (17) and (18), respectively:

$$
\begin{gathered}
v_{i d}^{(n+1)}=w \cdot v_{i d}^{(n)}+c_{1} \cdot \operatorname{rand} 1 \cdot\left(\text { pbest }_{i d}-x_{i d}^{(n)}\right)+c_{2} \cdot \operatorname{rand} 2 \cdot\left(\text { gbest }_{d}-x_{i d}^{(n)}\right) \\
x_{i d}^{(n+1)}=x_{i d}^{n}+v_{i d}^{(n+1)}
\end{gathered}
$$

(iv) Calculate the particle's fitness again and compare it with the particle's pbest. If the current value is better than pbest, then set the pbest value equal to the current value and the pbest location equal to the current location in $d$-dimensional space.

(v) Compare the fitness evaluation with the population's overall previous best. If the current value is better than gbest, then reset gbest to the current particle's array index and value.

(vi) Loop to step (iii) until a criterion is met, which is usually a sufficiently good fitness or a maximum number of iterations (generations). 
The flowchart of PSO is given in Fig. 10.

The definitions of the parameters are

$v_{i d}^{(n)}$ : velocity of individual $i$ at iteration $k, V_{d}^{\min } \leq v_{i d}^{(n)} \leq V_{d}^{\max }$,

$w$ : inertia weight factor,

$c_{1}, c_{2}$ : acceleration constants,

rand $_{1}$, rand 2 : uniform random numbers between 0 and 1 ,

$x_{i d}^{(n)}$ : current position of individual $i$ at iteration $n$,

pbest $t_{i}$ : pbest of individual $i$,

gbest: gbest of the group.

\subsection{Grey-based particle swarm optimization}

When the fittest particle gbest is regarded as the reference sequence and all particles $X$ are viewed as comparative values, grey relational analysis can be applied to analyze the similarity between them. Then both the inertia weight and acceleration coefficients of a specific particle are determined according to the corresponding relational grade. Since the result of grey relational analysis may differ for different generations, the algorithm parameters vary over the generations. According to Ref. 11, the grey relational coefficients between the fittest particle gbest and the $i$ th particle $X_{i}$ at the $d$ th dimension can be rewritten as

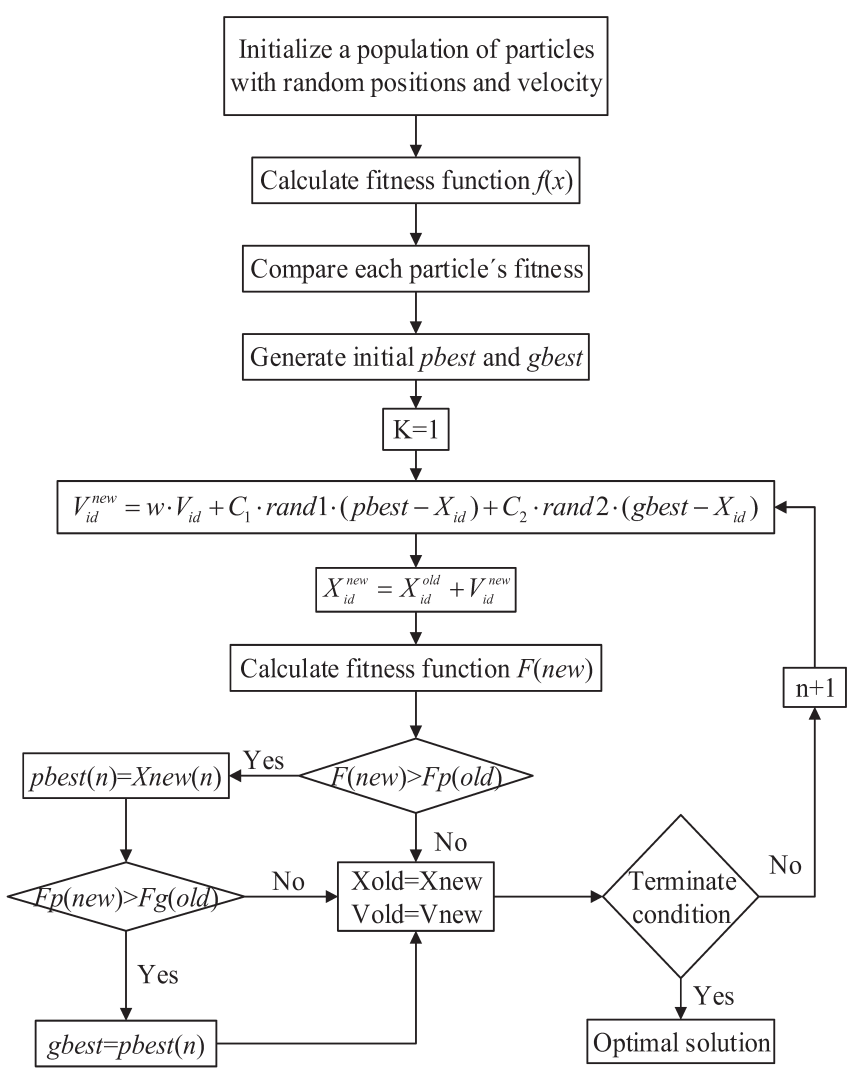

Fig. 10. Flowchart of PSO. 


$$
r_{i d}=r\left(\text { gbest }_{d}, x_{i d}\right)=\frac{\Delta_{\min }+\xi \cdot \Delta_{\max }}{\Delta_{i d}+\xi \cdot \Delta_{\max }},
$$

where $\Delta_{i d}=\mid$ gbest $_{d}-x_{i d} \mid, \Delta_{\max }=\max _{i} \max _{d} \Delta_{i d}, \Delta_{\min }=\min _{i} \min _{d} \Delta_{i d}$, and $\xi \in(0,1]$. Then the corresponding relational grade is given as

$$
g_{i}=g\left(\text { gbest }, X_{i}\right)=\sum_{d=1}^{D} r_{i d} / D
$$

\subsection{Adaptive weight particle swarm optimization}

Here, the outset particles are randomly divided into two groups with inertial weights of 0.9 and 0.4 . These two groups are independently searched. The function characteristic is determined by comparing the fitnesses of these two groups. Then, the two groups are merged for a suitable inertia weight of the characteristics, and the search is continued for half of the total time. Finally, a linear decreasing mode is employed for the rest of the time to improve the accuracy. ${ }^{(10)}$ The steps of AWPSO are as follows.

(i) Particles are randomly divided into two groups. One group uses an inertial weight of 0.9 $\times$ search speed and the other uses an inertial weight of $0.4 \times$ search speed.

(ii) When one-quarter of the generations are determined, merge the two groups using suitable inertia weights in the calculation until half the generations are determined.

(iii) Return to the linear decreasing mode.

Since the inertia weight is small, the particles will converge rapidly. However, premature convergence to a local optimal solution may occur. If the initial values rapidly converge to near the local minimum at the beginning, then a higher speed can be applied, which is very helpful in improving the accuracy and convergence rate.

\section{Experimental Results}

The controller in this study is designed by the C code composer on the TMS320C6713 DSP. In the DSP hardware structure, the JTAG connector is responsible for receiving and transmitting serial data, as shown in Fig. 11.

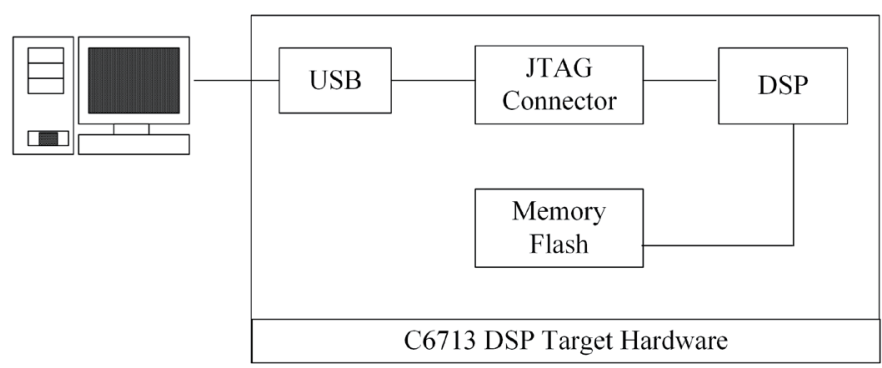

Fig. 11. DSP hardware structure. 
Figures $12-15$ show the results obtained by using FSMC-PID with the AWPSO controller. The controller can successfully guide the aircraft flying through wind shear of $58 \mathrm{ft} / \mathrm{s}$. When the wind speed is $58 \mathrm{ft} / \mathrm{s}$, the horizontal position at touchdown is $901.2 \mathrm{ft}$, the horizontal speed is $234.6 \mathrm{ft} / \mathrm{s}$, the vertical speed is $-1.9 \mathrm{ft} / \mathrm{s}$, and the pitch angle is $-9.9^{\circ}$.

FSMC-PID with the GPSO controller can also successfully guide the aircraft flying through wind shear of $58 \mathrm{ft} / \mathrm{s}$. When the wind speed is $58 \mathrm{ft} / \mathrm{s}$, the horizontal position at touchdown is $924.6 \mathrm{ft}$, the vertical speed is $-1.9 \mathrm{ft} / \mathrm{s}$, and the pitch angle is $-9.9^{\circ}$. Table 3 shows the results of using FSMC with different adjustment methods. The proposed controllers have superior performance to the conventional PID controller in the case of severe wind shear.

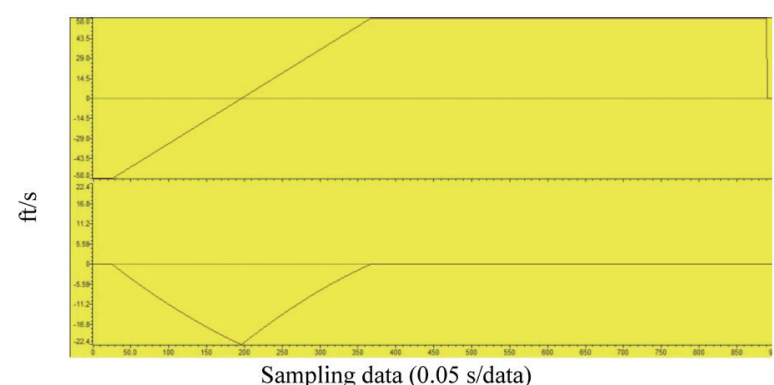

Fig. 12. (Color online) Wind shear profile $(58 \mathrm{ft} / \mathrm{s})$.

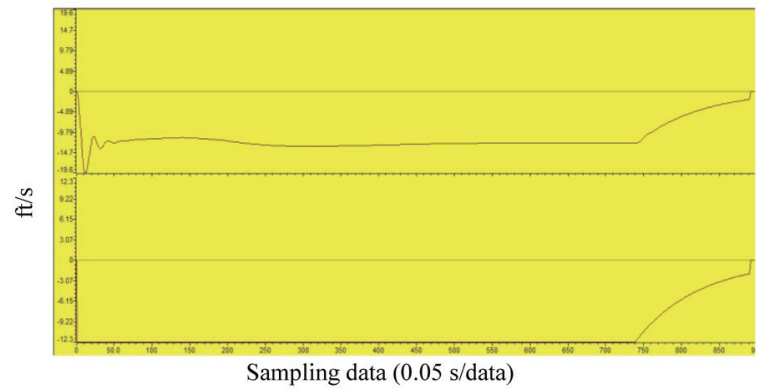

Fig. 14. (Color online) Vertical velocity and command.

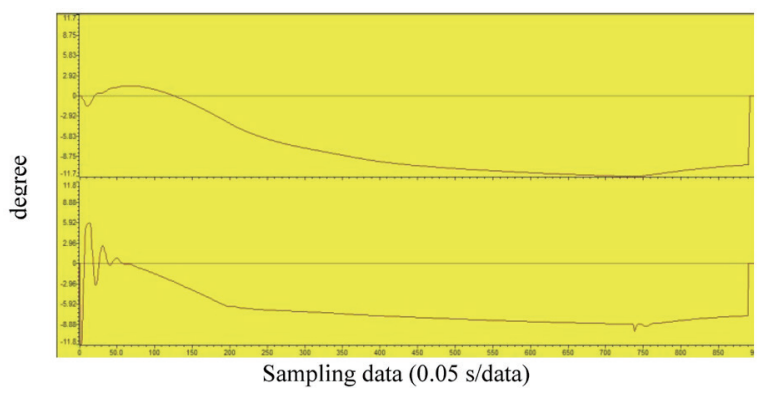

Fig. 13. (Color online) Aircraft pitch and pitch command.

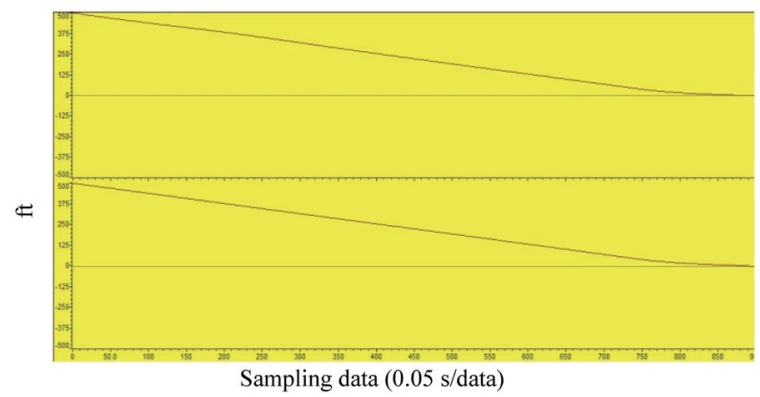

Fig. 15. (Color online) Aircraft altitude and command.

Table 3

Results of using FSMC with different adjustment methods.

\begin{tabular}{lcccc}
\hline Method & Wind speed $(\mathrm{ft} / \mathrm{s})$ & Landing point $(\mathrm{ft})$ & Aircraft vertical speed $(\mathrm{ft} / \mathrm{s})$ & Pitch angle $\left({ }^{\circ}\right)$ \\
\hline AWPSO & 58 & 901.2 & -1.9 & -9.9 \\
GPSO & 58 & 924.6 & -1.9 & -9.9 \\
PID & 11 & 995.1 & -2.4 & -2.9 \\
\hline
\end{tabular}




\section{Conclusions}

We proposed a DSP chip for designing embedded fuzzy SMC with optimization algorithms for an ALS. By comparison with software simulations, the DSP controller demonstrated the reliability of the proposed intelligent control system. All signals were sensed by relative sensors and sent to the DSP in our hardware simulation. In addition to the hardware implementation, we showed that the PSO method and intelligent controller improve the aircraft landing performance. FSMC compensation with a simple structure for a PID intelligent controller with optimization algorithms increases the efficiency of the controller, and the learning rules reduce its memory use. Our experimental results showed that the DSP controller is suitable for real-time online control. Its performance is similar to that of software simulation, and it can tolerate a wider range of wind shear conditions than the airplanes using previous models. The proposed control scheme can overcome wind shear of $58 \mathrm{ft} / \mathrm{s}$ and has superior performance to conventional PID control.

\section{References}

1 Aircraft Accident Statistics: http://www.planecrashinfo.com/cause.htm (accessed April 2020).

2 Federal Aviation Administration: Automatic Landing Systems (1971) AC 20-57A.

3 H. Buschek and A. J. Calise: J. Guidance, Control, and Dyn. 20 (1997) 42.

4 M.G. Cooper: Ph.D. Dissertation, Genetic Design of Rule-Based Fuzzy Controllers (University of California, Los Angeles, 1994)

5 Y. Iiguni, H. Akiyoshi, and N. Adachi: IEEE Trans. Aerosp. Electron. Syst. 34 (1998) 877.

6 H. Izadi, M. Pakmehr, and N. Sadati: Proc. IEEE Int. Conf. Control Applications (2003) CD-000202.

7 C. C. Jorgensen and C. Schley: Neural Networks for Control, W. T. Miller, R. S. Sutton, and P. J. Werbos, Eds. (The MIT Press, Cambridge, 1991) Chap. 17.

8 J. G. Juang, and J. Z. Chio: Int. J. Syst. Sci. 36 (2005) 77.

9 S. M. B. Malaek, N. Sadati, H. Izadi, and M. Pakmehr: Proc. IEEE 5th Control Conf. (2004) 365-373.

10 W. P. Lee: Master Thesis (Chung Yuan Christian University, Taiwan, 2007).

11 M. F. Yeh, M. S. Leu, K. C. Chang, and K. M. Chen: Proc. 2012 IEEE Int. Conf. Computer Science and Automation Engineering (2012) 189-193.

12 Sliding Mode Control, http://w3.cyu.edu.tw/control/ download/SMC3.pdf (accessed January 2016).

13 N. E. Sadr and H. R. Momeni: Proc. 2011 IEEE GCC Conf. and Exhibition (2011) 453-456.

14 J. T. Dai and J. G. Juang: J. Intell. Fuzzy Syst. 36 (2019) 1287.

15 J. G. Juang, H. K. Chiou, and C. L. Lee: J. Mar. Sci. Technol. 25 (2017) 442.

16 J. G. Juang and C. L. Lee: J. Univ. Computer Sci. 15 (2009) 2586.

17 Flight Safety Foundation-Taiwan: http://www.flightsafety. org.tw/news1.php?Code=1\&main_id=3\&pages=5 (accessed March 2016).

18 J. Kennedy and R. C. Eberhart: Proc. of IEEE Int. Conf. Neural Networks (1995) 1942-1948. 